\title{
Signal multiplexing in neural circuits - the superior colliculus deserves a new look
}

\author{
Michael Campos ${ }^{1 t}$ and MarkA. Segraves ${ }^{2 *+}$ \\ 1 Department of Neurosurgery, Massachusetts General Hospital/Harvard Medical School, Boston, MA, USA \\ Department of Neurobiology and Physiology, Northwestern University, Evanston, IL, USA \\ *Correspondence: m-segraves@northwestern.edu \\ ${ }^{\dagger}$ Michael Campos and Mark A. Segraves have contributed equally to this article.
}

The primate superior colliculus (SC) is a key component of the system controlling voluntary movements of the eyes and head. A longstanding view is that the SC maintains a retinotopic representation of visual space, and issues commands specifying movements referenced to the fovea. While an accurate representation of target position relative to the fovea is crucial to gaze shifts, information about the position of the eyes in their orbits is important to a number of potential SC functions that include determining the relative contributions of eye and head movements to generate gaze movements, integrating multimodal sensory input, and generating sequences of multiple movements. Nevertheless, the existence and potential role for eye position signals in the SC remains controversial.

Adding to a growing body of evidence for an eye position role in SC function, a recent paper by Groh (2011) makes clever use of microstimulation to demonstrate an effect of orbital position of the eyes on the magnitude of saccades evoked from the monkey SC. Although earlier reports revealed an eye position dependency for electrically evoked saccades in both cats and monkeys (Roucoux et al., 1980; Segraves and Goldberg, 1992; Cowie and Robinson, 1994; Freedman et al., 1996; MartinezTrujillo et al., 2003), whether the effects of stimulation implied the presence of an eye position signal in the SC remained unclear. Groh's report is significant because it compares the effects of two different stimulation frequencies, yielding greater insight into possible mechanisms, and provides a theoretical framework to help explain the presence and purpose of an eye position signal in the SC.

Groh's results suggest that an eye position signal modulates the readout of saccade commands issued from the SC. The effect of the eye position signal was greatest when low frequency stimulation was delivered, and weaker, though still present at slightly higher stimulation frequencies. Some early reports utilized still higher stimulation frequencies, such as $500 \mathrm{~Hz}$ (Robinson, 1972; Schiller and Stryker, 1972). Consistent with the trend observed by Groh, at these higher frequencies the effect of eye position is weaker still or entirely absent. While stimulation effects are notoriously difficult to interpret, Groh argues that these results confirm the existence of an eye position signal in the SC, and she further argues that the eye position signals should be incorporated into an accurate model of saccade generation. Two variations of a revised saccade generation model are proposed. Both acknowledge the existence of an eye position signal within the SC, but they differ in three ways: (1) how the putative eye position signal relates to well-characterized saccade vector signals in the same area, (2) whether that signal is enhanced or diminished by electrical stimulation, and finally (3) how and where that eye position signal is utilized downstream. In the first model (shown in Figure 10 of Groh), the direction of eye movement sensitivity is anti-correlated with the direction of saccade vector tuning in the same area. The electrical stimulation enhances the eye position signal. This signal is then $s u b$ tracted from (or ignored by) the subsequent stage of eye movement processing in a "normalization" step, and then added back at a later stage. Because of the anti-correlation, the result is a shorter than expected saccade. In the second model (Figure 11), the eye movement sensitivity is positively correlated with saccade vector tuning in the same area. Electrical stimulation serves to "clamp" or block the existing eye movement signal. Downstream areas then attempt to decode a signal that is lacking the expected eye position component. Because of the positive correlation, the end result is again a shorter saccade.
Seemingly conflicting physiological reports provide evidence in support of both of these models. Paré and Munoz (2001) found that eye position sensitivity was anti-correlated with the directional selectivity of co-present saccade vector signals in support of the first model. In support of the second model, Van Opstal et al. (1995) and Campos et al. (2006) found that eye position sensitivity was positively correlated with saccade vector tuning. However, as discussed in Groh (2011), these studies utilized different tasks. The analysis intervals used may have also contributed to the conflicting findings. Importantly, Paré and Munoz (2001) analyzed buildup activity that occurred close to the beginning of a saccade, in a task in which the monkeys were expected to have a strong re-centering bias. By continuously measuring the strength of saccade vector and eye position tuning, Campos et al. (2006) found that eye position sensitivity was strongest at times far removed from the saccade, and that the eye position signal strength decreased as the saccade onset approached. The natural scanning paradigm in the latter study may also have diminished the strength of a re-centering bias. Future physiological studies will be needed to characterize the strength of eye position signals in various task conditions.

Just as the physiological literature does not definitively resolve differences between the models offered by Groh, neither does the current understanding of the effect of electrical stimulation. If stimulation serves to enhance an already present eye position signal, the model shown in Figure 10 is supported. If stimulation, instead, serves to suppress the normally present eye movement signal, then the model shown in Figure 11 is supported. There is a growing body of evidence that electrical stimulation can either facilitate or suppress activity depending on the location of the stimulation, 
stimulus parameters including frequency, and current behavioral state (for a review, see Johnson et al., 2008).

To fully understand the stimulation results, it is critical to consider the additional effects of low frequency stimulation that were reported by Groh. At eccentric initial eye positions, saccades were evoked less reliably and usually at a longer latency. This indicates that Groh's study investigated the effects of electrical stimulation near the threshold at which eye movements could be evoked. Investigating this threshold, Stanford et al. (1996) demonstrated similar effects by varying stimulation frequencies at a fixed initial eye position, with more pronounced effects at lower frequencies close to the threshold. Groh, instead, varied the initial eye position, while using a fixed stimulation frequency and current, and achieved the same trend. This suggests that changes in initial eye position effectively modify the threshold at which saccades are evoked. It will be fruitful to characterize these effects by finely varying both the initial eye position and the stimulation frequencies and current, to understand whether and how eye position affects the threshold at which a saccade can be evoked.

Groh's first model posits that when firing rates are very high, there is a "normalization" process that occurs. When normalization does not occur individual spikes can be thought of as contributing a vote for a given eye movement vector. At higher frequencies the spikes are normalized such that the votes are submitted as a percentage of the total. This mechanism can account for the precise specification of saccade direction and magnitude at each SC site. That is, very high frequencies of activity do not produce increasingly larger magnitude saccades (although they do produce shorter latency, more reliable saccades). A normalization step is considered unnecessary for the second model (Figure 11). We propose, however, that the stimulation frequencies at which the normalization process is triggered should be considered a second threshold, in addition to the threshold at which a saccade is evoked. Once the normalization "threshold" is crossed, the effects of eye position on the saccade vector readout are strongly inhibited. Therefore, a normalization process could remain in the second model proposed by Groh, understanding that this mechanism is not invoked at the stimulation frequencies tested in this study.

Even with additional data points to characterize the thresholds in the SC and downstream, electrical stimulation will always remain difficult to interpret. Advances in novel neural stimulation techniques, notably optical stimulation of virally infected subpopulations, could yield even greater insight into the effects of stimulation. For instance, one could target specific subpopulations within the SC (including restricting stimulation to only neurons that project to or receive input from designated anatomical regions such as the NPH). Further, optogenetics includes tools with which to compare the effects of both excitation and inhibition on the metrics of an evoked saccade (Boyden et al., 2005; Diester et al., 2011).

The influences of eye position signals reported by Groh have important implications for some of the most fundamental questions in neuroscience. First, they provide new evidence that the SC contains neural circuitry capable of coordinating action plans (e.g., of the eye and head). Further study could reveal general mechanisms of effector coordination, similar to eye-hand coordination, or perhaps even sharing similarities with mechanisms of perceptual binding in sensory systems. Second, considering that primates generate two to four saccades per second in normal eye movement behavior, and considering the potential usefulness of an eye position signal for the planning of eye movement sequences, further study of the eye position signal in SC could yield insights into how sequences of movements are arranged in natural behavior. Third, by carrying multiple signals in the same neural substrate, including saccade vector, eye position, and signals relating to cognitive factors, the SC might reveal general principles by which information is multiplexed within neural circuits.

A representation of current eye position is just one of the many signals that converge on the SC. Given the simplicity of the saccade vector map in SC, and the body of work that has established its function and anatomical connections, it is an ideal structure to understand the subtle effects of a second type of signal as it modifies the dominant saccade vector command signal. This critical sensorimotor structure may be ideal for general studies of how different signals are combined or efficiently juggled in neural circuits (Stein and Meredith, 1993).

Groh's article taken another way suggests a form of frequency-division multiplexing in the SC. Whereas high frequencies may convey an eye-movement signal in purely retinotopic coordinates, lower frequencies might approach the "carrier frequency" of the eye position signal. In support of this idea, Corneil et al. (2007) have shown that low frequency stimulation can produce head movements without eliciting eye movements. That is, "sub-threshold" frequencies (below the threshold at which saccade are evoked and therefore encoded) might carry or encode pure head-movement signals. The tantalizing possibility is that the threshold at which a saccade is evoked is really more of a transition between these two frequency bands that carry signals to the different effectors. Pursuing this thread still further, the data from Campos et al. (2002) suggest a form of time-division multiplexing within the SC. In natural scanning of images, the time around the saccades were found to encode a purely retinotopic signal, but during fixation intervals, other relevant signals, such as eye position were expressed in the available bandwidth. Subthreshold stimulation to the SC has also been shown to modulate cognitive factors, such as attention (Cavanaugh and Wurtz, 2004), presumably through interactions with upstream areas.

One of the main attractions to the study of the eye movement system, and the SC in particular, has been its perceived simplicity. The oculomotor system has a relatively small number of moving parts, the eyes are light and easy to move, and the topographic map of eye movement vectors is elegantly arranged. With the growing evidence that the SC carries a diversity of signals, that it participates in cognitive functions in conjunction with upstream areas, and influences the coordination of multiple effectors with downstream areas, the attraction of the SC for future years may, instead, stem from an appreciation of its complexity.

\section{REFERENCES}

Boyden, E. S., Zhang, F., Bamberg, E., Nagel, G., and Deisseroth, K. (2005). Millisecond-timescale, genetically targeted optical control of neural activity. Nat. Neurosci. 8, 1263-1268.

Campos, M., Cherian, A., and Segraves, M. A. (2002). Spike triggered averaging of natural scanning reveals the dynamics of multiplexed eye and head signals in 
the primate superior colliculus. Soc. Neurosci. Abstr. Program No. 11.1.

Campos, M., Cherian, A., and Segraves, M. A. (2006). Effects of eye position upon activity of neurons in macaque superior colliculus. J. Neurophysiol. 95, 505-526.

Cavanaugh, J., and Wurtz, R. H. (2004). Subcortical modulation of attention counters change blindness. J. Neurosci. 24, 11236-11243.

Corneil, B. D., Munoz, D. P., and Olivier, E. (2007). Priming of head premotor circuits during oculomotor preparation. J. Neurophysiol. 97, 701-714.

Cowie, R. J., and Robinson, D. L. (1994). Subcortical contributions to head movements in macaques. I. Contrasting effects of electrical stimulation of a medial pontomedullary region and the superior colliculus. J. Neurophysiol. 72, 2648-2664.

Diester, I., Kaufman, M. T., Mogri, M., Pashaie, R., Goo, W., Yizhar, O., Ramakrishnan, C., Deisseroth, K., and Shenoy, K.V. (2011). An optogenetic toolbox designed for primates. Nat. Neurosci. 14, 387-397.

Freedman, E. G., Stanford, T. R., and Sparks, D. L. (1996). Combined eye-head gaze shifts produced by electrical stimulation of the superior colliculus in rhesus monkeys. J. Neurophysiol. 76, 927-952.

Groh, J. M. (2011). Effects of initial eye position on saccades evoked by microstimulation in the primate superior colliculus: implications for models of the SC read-out process. Front. Integr. Neurosci. 4:130. doi: $10.3389 /$ fnint.2010.00130

Johnson, M.D., Miocinovic, S., McIntyre, C. C., and Vitek, J. L. (2008). Mechanisms and targets of deep brain stimulation in movement disorders. Neurotherapeutics 5, 294-308.

Martinez-Trujillo, J. C., Klier, E. M., Wang, H., and Crawford, J. D. (2003). Contribution of head movement to gaze command coding in monkey frontal cortex and superior colliculus. J. Neurophysiol. 90, 2770-2776.

Paré, M., and Munoz, D. P. (2001). Expression of a recentering bias in saccade regulation by superior colliculus neurons. Exp. Brain Res. 137, 354-368.

Robinson, D. A. (1972). Eye movements evoked by collicular stimulation in the alert monkey. Vision Res. 12, 1795-1808.

Roucoux, A., Guitton, D., and Crommelinck, M. (1980). Stimulation of the superior colliculus in the alert cat. II. Eye and head movements evoked when the head is unrestrained. Exp. Brain Res. 39, 75-85.

Schiller, P. H., and Stryker, M. (1972). Single-unit recording and stimulation in superior colliculus of the alert rhesus monkey. J. Neurophysiol. 35, 915-924.

Segraves, M. A., and Goldberg, M. E. (1992). "Properties of eye and head movements evoked by electrical stimulation of the monkey superior colliculus," in The Head-Neck Sensory-Motor System, eds A. Berthoz, W. Graf, and P. P. Vidal (New York: Oxford University Press), 292-295.

Stanford, T. R., Freedman, E. G., and Sparks, D. L. (1996). Site and parameters of microstimulation: evidence for independent effects on the properties of saccades evoked from the primate superior colliculus. J. Neurophysiol. 76, 3360-3381.

Stein, B. E., and Meredith, M. A. (1993). The Merging of the Senses. Cambridge, MA: MIT Press.

Van Opstal, A. J., Hepp, K., Suzuki, Y., and Henn, V. (1995). Influence of eye position on activity in monkey superior colliculus. J. Neurophysiol. 74, 1593-1610.

Received: 11 March 2011; accepted: 21 March 2011; published online: 31 March 2011.

Citation: Campos M and Segraves MA (2011) Signal multiplexing in neural circuits - the superior colliculus deserves a new look. Front. Integr. Neurosci. 5:5. doi: 10.3389/ fnint.2011.00005

Copyright $(\odot) 2011$ Campos and Segraves. This is an openaccess article subject to a non-exclusive license between the authors and Frontiers Media SA, which permits use, distribution and reproduction in other forums, provided the original authors and source are credited and other Frontiers conditions are complied with. 